\title{
Multiband CCD Photometry of BL Lacertae
}

\author{
Omar M. Kurtanidze ${ }^{1}$ and Gotthard M. Richter \\ Astrophysikalisches Institute Potsdam, An der Sternwarte 16, D-14482, \\ Germany \\ Maria G. Nikolashvili \\ Abastumani Observatory, 383762 Abastumani, Republic of Georgia
}

\begin{abstract}
We present CCD opbservations of BL Lacertae obtained from August 1997 to August 1998. The maximum amplitudes in BVRI bands during observing period included most part of great ouburst and second one are equal to $3.00(\mathrm{rms}=0.03), 2.71(0.02), 2.53(0.01)$ and to $1.25(0.040), 1.11(0.03), 1.02(0.01) 0.91(0.03)$, respectively. The amplitude for variability is larger toward the blue end of the spectrum.
\end{abstract}

\section{Introduction}

BL Lacertae was discovered in 1929 by Guno Hoffmeister, who found it to vary by more than a factor two in one week and classified it as a short period variable star (Hoffmeister et al. 1990). Its true extragalactic nature was determined when Miller (Stickel et al. 1991) found faint emission lines at a redshift of 0.069 . Historically, BL Lac is known to show $\sim 5$ magnitude variation in optical band with episodic outbursts (Fan et al. 1998). During outbursts it showed a very strong activity including intra-night ones (Nikolashvili et al. 1999). The strong activity was also detected in the radio, X-ray and $\gamma$-ray bands.

\section{Observations}

We are monitoring BL Lacertae at Abastumani Observatory since August 4, 1997. All observations presented here were obtained with $70-\mathrm{cm}$ meniscus telescope and CCD Camera ST-6 $(375 \times 342,23 \times 27 \mu \mathrm{m})$ attached to the Newtonian focus (Kurtanidze and Nikolashvili 1999). During this period more than 3700 frames were obtained, including the frames obtained to study the Intraday variability. The exposure times varied from 60 to $180 \mathrm{sec}$ depending on the brightness of the object and the filter used. Instrumental magnitudes were obtained using DAOPHOT II routines (Stetson 1987). They were calibrated utilizing the sequances of comparison stars $\mathrm{C}$ and $\mathrm{H}$ of Smith et al. (1985).

\footnotetext{
${ }^{1}$ Visiting Astronomer, on leave from Abastumani Observatory
} 


\section{Results}

The preliminary results of observations of BL Lac during great summer 1997 outburst are presented by Blazar Monitoring Groups at the Annual OJ-94 Meeting (Takalo et al. 1998). Detailed results of the summer 1997 multiband campaigns are published by Webb et al. (1988), Sobrito et all. (1999) and Tosti et al. $(1999 a, b)$. We present here observations carried out during great outburst and after great outburst, when BL Lac possesed the second outburst. Most of the frames were obtained in $\mathrm{R}$ band.

The maximum variation is observed in $\mathrm{B}$ band and equals to 3.00 ( $\mathrm{rms}=0.03$ ) and $1.25 \mathrm{mag}(0.04)$. The variations in the VRI bands are within $2.71(0.02)$, $2.53(0.01)$ and $1.11(0.03), 1.02(0.01), 0.91(0.03)$ respecctively. This means that variations are larger at shorter wavelength or the object become bluer in the active phase.

Acknowledgments. We thank the SOC for the kind invitation and financial support. O.M.K. grateffully acknowledges invaluable financial support of the Astrophisikalisches Institut Potsdam without which this Programm will never be conducted.

\section{References}

Fan J.H., Xie G.Z., Pecontal E., et al., 1999, ApJ507, 173

Hoffmeister G., Richter G., Wenzel W., 1990, Veranderlichte Sterne, Johann Ambrisius Barth, Leipzig

Kurtanidze O.M., Nikolashvili M.G., Takalo L. O., 1998, in: Tosti G., Takalo L. (eds.) Proc. OJ-94 Annual Meeting 1997, Multifrequency Monitoring of Blazars. Pubblicazioni Osservatorio Astronomico Università di Perugia, Perugia, vol. 3, p. 193

Kurtanidze O.M., Nikolashvili M.G., 1999, in: Raiteri C.M., Villata M. \& Takalo L.O (eds.) Proc. OJ-94 Annual Meeting 1999, Blasar monitoring towards the third millennium. Osservatorio Astronomico di Torino, p.25

Nikolashvili M.G., Kurtanidze O.M., Richter G.M., 1999 in: Raiteri C.M., Villata M. \& Takalo L.O (eds.) Proc. OJ-94 Annual Meeting 1999, Blasar monitoring towards the third millennium. Osservatorio Astronomico di Torino, p.36

Smith P.S., Balonek T.J., Heckert P.A., et al., 1985, AJ90, 1184

Sobrito G., Villata M., Raiteri C.M., 1999, Blazar Data v.1, no.5

Stetson P.B., 1987, PASP99, 191

Stickel M., Padovani P., Urry C.M., et al., 1991, ApJ374, 431

Takalo L.O., et al., 1998, in: Tosti G., Takalo L. (eds.) Proc. OJ-94 Annual Meeting 1997, Multifrequency Monitoring of Blazars. Pubblicazioni Osservatorio Astronomico Università di Perugia, vol. 3, p. 193

Tosti G., Lucciani M., Fiorucci M., et al., 1999, Blazar Data v.2, no.1

Webb J.R., Freedman I., Howard E., et al. 1998, AJ115, 2244 